\title{
Ninety-Fourth Annual Report of The American Phytopathological Society
}

\section{REPORT OF THE TREASURER}

The Financial Advisory Committee (FAC) and APS headquarters' staff members met 24 August 2001 in Salt Lake City and 25 October 2001 and 1 March 2002 in St. Paul, MN during FY0102 to review financial matters related to the operation of the Society. The notes from these meetings have been approved by FAC and notes of all meetings are on file at APS headquarters.

After allocations for maintenance reserves, operating reserves, and capital improvement expenses, FY01-02 was concluded with a surplus of $\$ 47,741$, which represents $1.2 \%$ of income; a very positive change after 3 years of expenses exceeding revenues. The deficits over the previous 3 years resulted from significant investment in our Society moving into the digital and electronic age. New software to enhance efficiency at headquarters was installed, we sought new alliances to enhance our bussiness operation, we have begun to move from a hardcopy perspective to an electronic perspective, our web presence has become conspicuous, and new initiatives to support research, education, extension, and public policy have been established. During this same period, author page charges were significantly reduced, dues are linked to the consumer price index and thus have minimally increased, and prices of other products are set to be as affordable as possible. Our primary source of income continues to be the high quality journals. Our renewed financial vigor comes primarily from containing expenses. Much of this containment of expenses is from the leadership provided by APS headquarters that works closely with the elected and appointed membership in a recurring self study and planning process. The 11 income and expense categories for the Society are detailed in Table 1. Our total income $(\$ 4,045,587)$ was derived from nine sources as indicated in Figure 1 , and our total operating expenses $(\$ 3,997,846)$ incurred during FY01-02 were partitioned as indicated in Figure 2.

The income and expenses of the Society for the most recent 10 FYs are presented in Table 2. The total assets of the Society as

TABLE 2. Comparison of The American Phytopathological Society fiscal years 1993 to 2002 before reserve allocation

\begin{tabular}{lccc}
\hline Fiscal year & Income & Expenses & Surplus (deficit) \\
\hline FY02 & $\$ 4,045,587$ & $\$ 3,997,846$ & $\$ 47,741$ \\
FY01 & $\$ 3,952,594$ & $\$ 4,059,101$ & $(\$ 106,507)$ \\
FY00 & $\$ 3,649,863$ & $\$ 3,724,910$ & $(\$ 75,047)$ \\
FY99 & $\$ 3,662,093$ & $\$ 3,692,259$ & $\$ 30,166)$ \\
FY98 & $\$ 3,589,253$ & $\$ 3,431,727$ & $\$ 157,526$ \\
FY97 & $\$ 3,418,515$ & $\$ 3,216,088$ & $\$ 202,427$ \\
FY96 & $\$ 3,198,990$ & $\$ 2,974,105$ & $\$ 224,885$ \\
FY95 & $\$ 3,152,468$ & $\$ 2,907,285$ & $\$ 245,183$ \\
FY94 & $\$ 3,062,160$ & $\$ 2,902,793$ & $\$ 159,367$ \\
FY93 & $\$ 2,843,296$ & $\$ 2,574,006$ & $\$ 269,290$ \\
\hline
\end{tabular}

TABLE 1. Audited summary of income and expenses as related to function of The American Phytopathological Society

\begin{tabular}{|c|c|c|c|c|c|c|c|c|c|}
\hline \multirow[b]{2}{*}{ Function } & \multicolumn{4}{|c|}{ Income } & \multicolumn{4}{|c|}{ Expenses } & \multirow[b]{2}{*}{ Profit (loss) } \\
\hline & Actual & $\%$ & Budget & $\%$ & Actual & $\%$ & Budget & $\%$ & \\
\hline Member services & $\$ 305,234$ & $8 \%$ & $\$ 306,500$ & $7 \%$ & $\$ 581,704$ & $15 \%$ & $\$ 601,723$ & $14 \%$ & $(\$ 276,470)$ \\
\hline Short course & $\$ 15,613$ & $0 \%$ & $\$ 4,500$ & $0 \%$ & $\$ 14,848$ & $0 \%$ & $\$ 17,264$ & $0 \%$ & $\$ 765$ \\
\hline Plant Disease & $\$ 656,333$ & $16 \%$ & $\$ 632,750$ & $15 \%$ & $\$ 360,171$ & $9 \%$ & $\$ 375,687$ & $9 \%$ & $\$ 296,162$ \\
\hline Phyto News & $\$ 9,334$ & $0 \%$ & $\$ 3,800$ & $0 \%$ & $\$ 62,724$ & $2 \%$ & $\$ 73,081$ & $2 \%$ & $(\$ 53,390)$ \\
\hline MPMI & $\$ 448,522$ & $11 \%$ & $\$ 417,200$ & $10 \%$ & $\$ 321,228$ & $8 \%$ & $\$ 315,517$ & $8 \%$ & $\$ 127,294$ \\
\hline Online products & $\$ 37,960$ & $1 \%$ & $\$ 26,600$ & $1 \%$ & $\$ 24,252$ & $1 \%$ & $\$ 30,401$ & $1 \%$ & $\$ 13,708$ \\
\hline $\mathrm{G} \& \mathrm{~A}^{\mathrm{a}}$ & $\$ 21,051$ & $1 \%$ & $\$ 32,100$ & $1 \%$ & $\$ 892,599$ & $21 \%$ & $\$ 909,682$ & $21 \%$ & $(\$ 871,548)$ \\
\hline $\begin{array}{l}\text { Total } \\
\text { Operating surplus (loss) }\end{array}$ & $\$ 4,045,587$ & $100 \%$ & $\$ 4,167,225$ & $100 \%$ & $\$ 3,997,846$ & $100 \%$ & $\$ 4,250,336$ & $100 \%$ & $\$ 47,741$ \\
\hline
\end{tabular}

${ }^{\mathrm{a}} \mathrm{G} \& \mathrm{~A}$ is assigned to functions based on payroll dollars.

Income

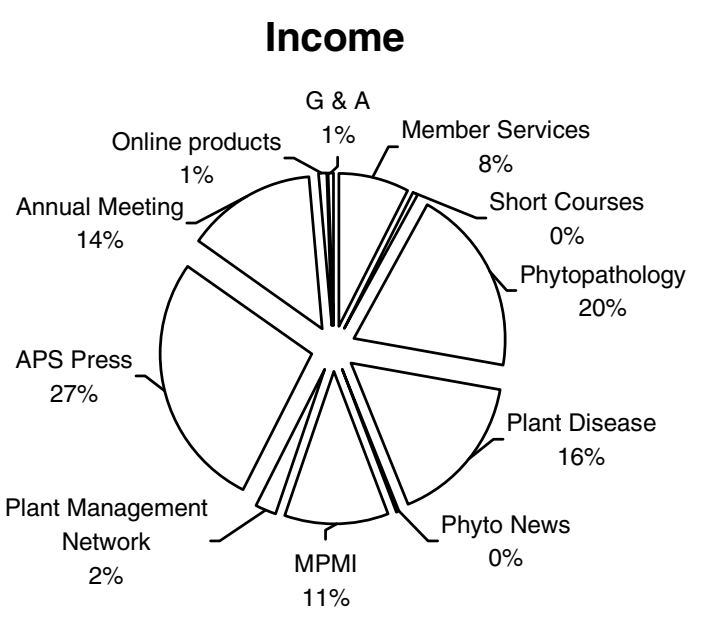

Fig. 1.

Publication no. P-2002-1112-010

\section{Expenses}

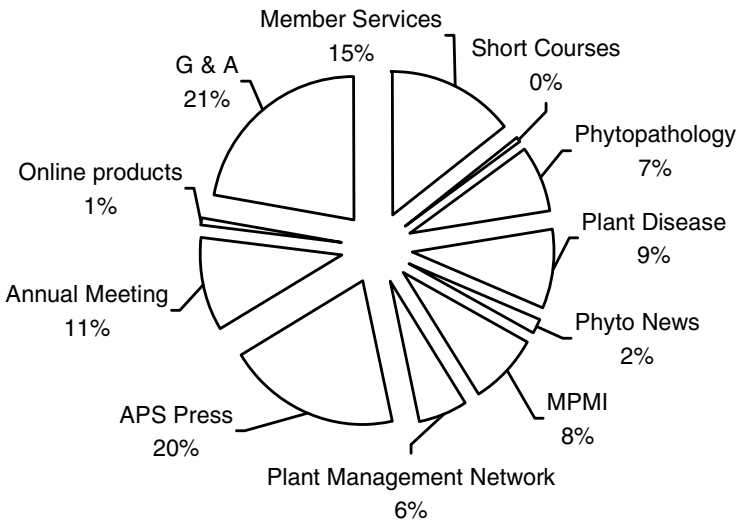

Fig. 2. 
of 30 June 2002, including restricted funds, were $\$ 4,949,167$ and current liabilities totaled $\$ 3,771,983$, producing a membership equity of $\$ 1,177,183$ that includes the FY01-02 surplus of $\$ 47,741$. During FY01-02, several initiatives continued under development to enhance member services and improve outreach that included the Plant Management Network, the Education

\section{REPORT OF THE AUDITOR}

The American Phytopathological Society has its accounts audited annually by a certified public accountant. In recent years, the accounting firm has been Lethert, Skwira, Schultz, \& Co., St. Paul, MN. Only the Balance Sheet and the Statement of Changes in Net Assets, as of 30 June 2002, are being published in detail. Any member wishing a copy of the full audit may obtain one on request.

\begin{tabular}{|c|c|c|c|c|}
\hline & & 2001 & & 2002 \\
\hline \multicolumn{5}{|l|}{ Assets } \\
\hline Cash (note 3) $)^{\mathrm{a}}$ & $\$$ & $1,251,883$ & $\$$ & $1,399,870$ \\
\hline Interest receivable & & 306 & & - \\
\hline Accounts receivable, net (notes 2 and 17) & & 203,914 & & 368,549 \\
\hline Unconditional promise to give & & - & & - \\
\hline Investments (note 4) & & $1,513,547$ & & $1,407,670$ \\
\hline Prepaid expenses (note 5) & & 346,766 & & 244,516 \\
\hline Inventory & & 753,924 & & 785,516 \\
\hline Prepaid pension (note 14) & & 75,781 & & 35,973 \\
\hline Property and equipment, net (note 6) & & 546,379 & & 521,934 \\
\hline Total assets & $\$$ & $4,692,500$ & & $4,764,048$ \\
\hline \multicolumn{5}{|l|}{ Liabilities and net assets } \\
\hline \multicolumn{5}{|l|}{ Liabilities } \\
\hline Accounts payable (note 17 ) & $\$$ & 247,226 & $\$$ & 272,770 \\
\hline Accrued pension expense & & 39,660 & & 56,860 \\
\hline Agency funds payable & & 10,931 & & 10,003 \\
\hline Deferred revenues (note 7) & & $1,110,356$ & & $1,238,302$ \\
\hline Long-term debt (note 8) & & 792 & & 4,000 \\
\hline Total liabilities & & $1,408,965$ & & $1,581,935$ \\
\hline \multicolumn{5}{|l|}{ Net assets } \\
\hline Unrestricted (note 9) & & $2,469,806$ & $\$$ & $2,381,838$ \\
\hline Temporarily restricted (note 10 ) & & 199,159 & & 104,767 \\
\hline Permanently restricted (note 11 ) & & 614,570 & & 695,508 \\
\hline Total net assets & & $3,283,535$ & & $3,182,113$ \\
\hline Total liabilities and net assets & $\$$ & $4,692,500$ & $\$$ & $4,764,048$ \\
\hline
\end{tabular}

a There are accompanying notes that are an integral part of this financial statement and are available upon request.
Center, and the increased use of reserves of the Society in supporting the APS Annual Meeting. Your recommendations related to the activities of APS should be expressed to members of the APS Council. The Treasurer can be contacted at sherwood@uga.edu. The audited financial statement for FY01-02 appears in this issue of Phytopathology.

The American Phytopathological Society statement of Changes in Net Assets Year ended 30 June 2002 (with comparative totals for 2001)

\begin{tabular}{|c|c|c|}
\hline & 2001 & 2002 \\
\hline \multicolumn{3}{|l|}{ Unrestricted net assets } \\
\hline \multicolumn{3}{|l|}{ Support and revenue } \\
\hline Federal financial assistance & $\$ \quad 25,371$ & 10,000 \\
\hline Contributions & 3,534 & 4,345 \\
\hline Membership dues & 285,741 & 275,199 \\
\hline Subscriptions & $1,345,576$ & $1,506,678$ \\
\hline Reprints and demand articles & 89,578 & 78,856 \\
\hline Abstracts & 20,795 & 36,320 \\
\hline Page charges & 156,655 & 182,497 \\
\hline Processing fees & 98,215 & 78,620 \\
\hline Back issues & 16,435 & 16,696 \\
\hline Color charges & 112,885 & 108,015 \\
\hline Advertising & 33,035 & 40,170 \\
\hline Books, slides, and CD-Roms & $1,289,099$ & $1,070,136$ \\
\hline Annual meeting & 392,553 & 564,567 \\
\hline Short courses & 39,419 & 15,603 \\
\hline Royalty income & 25,569 & 20,871 \\
\hline Investment income (note 4$)^{\mathrm{a}}$ & 95,959 & 48,272 \\
\hline Gain on sale of investments (note 4) & 34,607 & $(27,723)$ \\
\hline Unrealized gain (loss) on investments (note 4 ) & $(148,107)$ & $(89,804)$ \\
\hline Other & 31,567 & 17,662 \\
\hline Total revenue & $3,948,483$ & $3,956,990$ \\
\hline \multicolumn{3}{|l|}{ Net assets released from restrictions } \\
\hline Restrictions satisfied by payments & 61,053 & 106,408 \\
\hline \multicolumn{3}{|l|}{ Expenses } \\
\hline \multicolumn{3}{|l|}{ Program services } \\
\hline Member services & 617,399 & 594,139 \\
\hline Journals & $1,210,343$ & $1,273,244$ \\
\hline Books & $1,003,428$ & 804,653 \\
\hline Annual meeting & 403,058 & 530,297 \\
\hline Grants and awards & 25,786 & 36,850 \\
\hline Short c & 18,677 & 14,847 \\
\hline Online services & - & 24,251 \\
\hline Total program services & $3,278,691$ & $3,278,281$ \\
\hline \multicolumn{3}{|l|}{ Supporting services } \\
\hline Management and general & 841,653 & 870,851 \\
\hline Fundraising & (381) & 4,234 \\
\hline Total supporting services & 841,272 & 875,085 \\
\hline Total expenses & $4,119,963$ & $4,153,366$ \\
\hline Increase in unrestricted net assets & $(110,427)$ & $(89,968)$ \\
\hline \multicolumn{3}{|l|}{ Temporarily restricted net assets } \\
\hline Support for books & 48,855 & 14,426 \\
\hline Support for annual meeting socials & 5,400 & 21,450 \\
\hline Support fo & - & - \\
\hline Support for Virology Committe & 10,700 & 1,000 \\
\hline Support for student travel & 11,430 & 5,016 \\
\hline Investment income (note 4) & 20,981 & 14,740 \\
\hline Gain (loss) on sale of investments (note 4) & 3,852 & $(9,610)$ \\
\hline Unrealized gain (loss) on investments (note 4) & $(36,780)$ & $(33,006)$ \\
\hline Restrictions satisfied by payments & $(61,053)$ & $(106,408)$ \\
\hline Increase in temporarily restricted net assets & 3,385 & $(92,392)$ \\
\hline \multicolumn{3}{|l|}{ Permanently restricted net assets } \\
\hline Support for operations & 7,529 & 4,444 \\
\hline Support for student travel & 49,601 & 32,468 \\
\hline Support for student speaker & 10,075 & 20,285 \\
\hline Support for research & 1,340 & 3,478 \\
\hline Support for awards & 32,225 & 20,000 \\
\hline Unrealized loss on investments (note 4) & 591 & 263 \\
\hline Increase in permanently restricted net assets & 101,361 & $\begin{array}{r}80,938 \\
(101422)\end{array}$ \\
\hline Decrease in net assets & $(5,681)$ & $(101,422)$ \\
\hline Net assets, beginning of year & $3,289,216$ & $3,283,535$ \\
\hline Net assets, end of year & $\$ 3,283,535$ & $\$ 3,182,113$ \\
\hline
\end{tabular}

${ }^{a}$ There are accompanying notes that are an integral part of this financial statement and are available upon request. 